\title{
El tratamiento de la demencia con antipsicóticos atípicos aumenta el riesgo de muerte
}

Schneider L, et. al. JAMA. 2005 19;294(15):1934-43

\section{Objetivo}

Evaluar la mortalidad en pacientes con demencia tratados con antipsicóticos atípicos.

\section{Fuente y selección de datos}

Fueron buscados hasta abril de 2005 ensayos clínicos controlados y aleatorizados que incluyeran pacientes con demencia tratados con antipsicóticos atípicos comercializados en EEUU (aripiprazol, olanzapina, quetiapina, risperidona y ziprasidona) en MEDLINE, la base Cochrane de ensayos controlados, presentaciones en eventos científicos hasta 2004 y en los laboratorios responsables de las drogas.

El efecto se analizó para cada droga individualmente y para el total como grupo, con subanánlisis en función de la publicación y del ámbito (ambulatorio vs. internación).

\section{Resultados principales}

De los 513 estudios encontrados, se describen en la tabla los que cumplieron los criterios de inclusión.

Salvo uno, que comparó olanzapina con risperidona, el resto fueron controlados contra placebo y no se encontraron estudios con ziprasidona.
Tabla. Resultados principales.

\begin{tabular}{|c|c|c|c|c|c|}
\hline $\begin{array}{c}\text { Droga (NT de } \\
\text { ensayos) }\end{array}$ & $\begin{array}{l}\text { Droga activa } \\
\text { Muertes/ "n" }\end{array}$ & $\begin{array}{l}\text { Placebo } \\
\text { Muertes/"n" }\end{array}$ & OR (IC95\%) & $\operatorname{RR}^{2}(1695 \%)$ & NND*(1695\%) \\
\hline Aripiprazol (3) & $21 / 603$ & $6 / 348$ & $21,73(0,70-4,30)$ & $1,99(0,86-4,42)$ & - \\
\hline Olanzapina (5) & 31/1/184 & $6 / 478$ & $1,91(0,79-4,59)$ & $2,31(1,00-5,35)$ & - \\
\hline Quetiapina (3) & $21 / 391$ & 7246 & $1,67(0,70-4,03)$ & $1,86(0,86-3,93)$ & - \\
\hline Risperidona (5) & 45/1175 & 222779 & $1,30(0,76-2,23)$ & $1,35(0,85-3,93)$ & - \\
\hline Total & $11833533(3,5 \%)$ & 41/1851 (2,3\%) & $1.54(1,06-2,23)$ & $1.65(1,1922,29)$ & $100(50-250)$ \\
\hline
\end{tabular}

\&En base a pacientes-año de exposición a drogas o placebo. *№ necesario para dañar

No se encontraron diferencias estadísticamente significativas en función del ámbito, la publicación o el abandono. Se observó un mayor riesgo de muerte en los pacientes expuestos a las drogas por breves períodos ( 8 a 12 semanas) y no hubo heterogeneidad* entre los resultados de los estudios, lo que sugeriría un fenómeno de clase. La modesta efectividad del tratamiento evidenciada en meta-análisis específicos (número necesario para tratar de 4 a 12 pacientes) hace que la probabilidad ayudar vs. dañar también sea modesta, siendo esperable globalmente una muerte por cada 9 a 25 pacientes beneficiados.

\section{Conclusiones}

Existe un riesgo aumentado de muerte en pacientes con demencia tratados con antipsicóticos atípicos.

\section{Comentario}

En pacientes con demencia, las conductas agresivas, alucinaciones y otros síntomas neuropsiquiátricos son frecuentes y de difícil manejo, con gran impacto sobre los cuidadores.

Desde los ' 80 se viene señalando el sobreuso de los neurolépticos para el manejo de estos síntomas, pese a ser una indicación fuera de prospecto ("off the label"). Con la aparición de los antipsicóticos atípicos, éstos rápidamente reemplazaron a los típicos por sus supuestos menores efectos secundarios y su mayor eficacia. Sin embargo, no existían ensayos que respaldasen esas percepciones. La evidencia de que aumentarían el riesgo de accidente cerebrovascular (ACV) proveniente de un ensayo clínico controlado con risperidona y olanzapina generó durante 2002 en Canadá un alerta sobre el uso de risperidona en ancianos por parte de las autoridades regulatorias y del laboratorio titular de la patente'. En 2003 fue adoptada igual medida por la (Administración de Drogas y Alimentos de EEUU: FDA) y extendida a otros antipsicóticos atípicos en los años siguientes. En abril de 2005 un comunicado alertó sobre el riesgo de aumento de la mortalidad global ${ }^{2}$.

A principios de 2005, investigadores canadienses publicaron una interesante investigación, que a partir de un estudio observacional, evidenciaba que el riesgo de aumento de ACV se encuentra pre- sente en todos los antipsicóticos, incluidos los típicos ${ }^{3}$.

Este estudio nos alerta sobre la importancia de insistir en la necesidad de realizar ensayos clínicos en poblaciones especiales (ancianos, niños, mujeres y minorías étnicas) ya que su exclusión de los ensayos clínicos las hacen aún más vulnerables; debido a que luego de introducidos los productos en el mercado y ante la falta de evidencia en estos subgrupos, los profesionales de la salud se ven obligados a extrapolar los resultados.

\section{Conclusiones del comentador}

La falta de otros tratamientos farmacológicos validados, no nos deja, en muchos casos, otra opción que medicar a estos pacientes con este grupo de fármacos, aún sabiendo que están "fuera de prospecto" y que condicionan un importante riesgo de muerte. Sin embargo, esta información nos obliga a realizar una evaluación cuidadosa de otras alternativas no farmacológicas, antes de indicarlos y, cuando no quede otra alternativa, a ser muy cautos con la dosis, duración del tratamiento y a la evaluación de otros factores de riesgo.

Carlos David Silva [ Médico Psiquiatra. Diplomado en Salud Pública (ESPUBA) Director de Equipo PSY, Adrogué, Buenos Aires. ]

Silva C. Tratamiento de demencia con antipsicóticos atípicos y riesgo de muerte. Evid. act. pract. ambul. 9(1):10.En-Febr.2006. Comentado de: Schneider LS, Dagerman KS, Insel P. Risk of death with atypical antipsychotic drug treatment for dementia: meta-analysis of randomized placebo-controlled trials. JAMA. 2005; 294(15):1934-43- PMID: 16234500.

Referencias

1. Health Canada Therapeutic Products Directorate, Risperdal. Carta de Alerta, October 11, 2002. disponible en: http://www.hc-sc.gc.ca/dhp-mps/medeff/advisories-avis/prof/2002/risperdal_hpc-cps_e.html. Acceso verificado el 9/12/2005.

2. FDA Public Health Advisory: deaths with antipsychotics in elderly patients with behavioral disturbances. Disponible en: http://www.fda.gov/cder/drug/advisory/antipsychotics.htm. Acceso verificado el 9/12/2005.

3. Atypical antipsychotic drugs and risk of ischaemic stroke: population based retrospective cohort study. Gill S., Rochon P, Herrmann N y otros. BMJ. 2005 Feb 26;330(7489):445. 